\title{
Current Development Status of a Particle Size Analyzer for Coated Particle Fuel
}

\author{
Andrew T. Nelson, John D. Hunn and Thomas P. Karnowski
}

\begin{abstract}
Work was performed to develop a prototype Particle Size Analyzer (PSA) for application to coated particle fuel characterization. This system was based on a light obscuration method and targeted towards high throughput analysis. Although never matured to the point of replacing existing lower throughput optical microscopy shadowgraph methods, the system was successfully applied to automating the counting of large particle samples for increased accuracy in calculating mean particle properties based on measurements of multiparticle samples. The measurement of particle size with the PSA was compared to current shadowgraph techniques and found to result in considerably greater throughput at the cost of larger measurement uncertainty. The current algorithm used by the PSA is more sensitive to particle shape and this is a likely cause of the greater uncertainty when attempting to measure average particle diameter. The use of the PSA to measure particle shape will require further development. Particle transport through the PSA and stability of the light source/detector are key elements in the successful application of this technique. A number of system pitfalls were studied and addressed.
\end{abstract}




\section{Introduction}

The Advanced Gas Reactor (AGR) Fuel Development and Qualification program is a United States Department of Energy (US-DOE) program to promote the technology required to produce an approved fuel form for the Very High Temperature Gas Cooled Reactor (VHTR), designated as the premier US-DOE Generation IV near-term reactor concept for the Next Generation Nuclear Plant (NGNP). The AGR fuel development effort involves recapturing methods for manufacturing and characterizing tri-isotropic (TRISO) coated uranium bearing kernels. A large number of these particles are then compacted into either a spherical or cylindrical shape for insertion into the reactor. The TRISO coatings are responsible for the enhanced safety of nuclear reactors fueled by this type of system, as all radioactive fission products remain quarantined within the coated particle. However, ensuring that this containment remains effective throughout a wide range of temperatures and material irradiation levels requires extensive testing and thorough characterization at all stages of production.

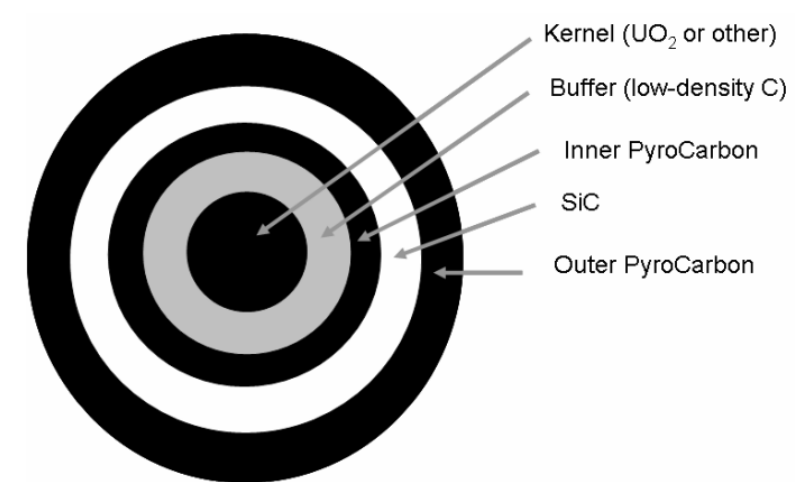

Figure 1. Diagram of TRISO fuel particle layers.

The specific coated particle variant initially investigated by the AGR program consisted of a $350 \mu$ m diameter uranium oxycarbide kernel (UCO) surrounded first by a layer of buffer carbon, then an inner pyrolytic carbon coating (IPyC), a hard silicon carbide layer (SiC) sheath, and finally an outer pyrolytic carbon layer (OPyC) (Figure 1). The four coatings on top of the kernel typically yield a finished particle diameter of roughly 800 $\mu \mathrm{m}$.

A need for state of the art characterization techniques within the AGR program has been recognized from the outset of research efforts and accordingly been pursued hand in hand with refinement of the manufacturing processes. Without the ability to completely scrutinize the geometric and chemical properties of a particle lot, correctly evaluating variations within a specific stage of the coating process becomes impossible. In addition to a number of other procedures developed to measure specific quantities, an automated shadowgraph technique using optical microscopy was implemented to meet the need for particle size and shape data for whole kernels or coated particles. ${ }^{1,2}$ Particle sizing is performed using an optical microscope and an automated stage which images single layer trays of backlit particles to produce darkened silhouettes. A Matlab algorithm then processes the stored image files. The program identifies individual particles and then calculates a perimeter of the particle based upon the transition along a particle's edge 
from dark pixels to light pixels. This perimeter data is then used to generate average particle diameter and aspect ratios to produce histograms representing the size and shape distribution of the particle lot. This procedure yields high quality data on particle size and shape, but is not used for counting since particles on the edge of images are omitted from analysis. The entire procedure requires two to three hours of technician time to prepare and image the particle trays, followed by up to twelve hours of CPU time for automated analysis.

The throughput limitations of the shadowgraph technique provided motivation to investigate other means to count and analyze whole particles. A high speed particle size analyzer (PSA) would serve multiple uses within the AGR characterization effort, both for simple particle counting and for more effective sampling of size and shape. The measurement of the average particle weight is an example of a procedure that would benefit from an improved particle counting method. The average particle weight is required for numerous calculations that are directly cited in the fuel specification. It is impractical to weigh individual particles, so five subsets on the order of one to two hundred particles are weighed and then the average particle weight is calculated from the subset weight divided by the number of particles in the subset. The uncertainty of the average particle weight can be reduced by increasing the size of the particle sample. However, increasing the sample size is impractical when using a manual counting method due to the effort involved and the likelihood of increased human error when manually counting larger samples. With a PSA, sample sizes of several thousand particles can be easily and accurately counted.

Given the appeal of a system which could improve upon the hand-counted particle process and the fact that the currently-employed optical imaging technique inspects individual particles rather than complete lots, expanding or improving the imaging and analysis system to output particle counts would be possible. However, the main drawback of pursuing this avenue lies in the long preparation times for each tray. Even if no modifications were necessary to the technician's procedure and only an adaptation of the algorithm was required to yield particle counts, each tray requires at least 20 minutes to assemble and image. Each tray holds about 500 TRISO particles (more for the smaller kernels), so improving the hand-counted particle lot quantities by two orders of magnitude would require more than 60 hours of data collection.

Despite these potential improvements in existing particle counting and size and shape characterization that a PSA may offer, the existing optical technique is sufficient to get the mean and standard deviation required to show that a specific particle lot meets the various property specifications for size and shape. A 'large' number of TRISO particles sized by optical analysis using multiple mounts was typically around 1500 . When kernels were imaged this number increased to about 4000 . These numbers were sufficient to calculate a mean and standard deviation to a high degree of confidence, but far too small to statistically analyze for low frequency $\left(10^{-4}-10^{-5}\right)$ attribute-type defects. Such analysis typically requires sample sizes of 100,000 or more and is only within reach of a system designed for such a purpose. 


\section{PSA Design}

The original design specification for the PSA called for the development of a nondestructive, high throughput device capable of sampling $100 \%$ of particles within the sample lot. A non-destructive method was necessary given the limited numbers and wide range of variations in particles produced by the program. Along the same lines, successful integration of the PSA into AGR fuel characterizations would require large numbers of particles processed weekly - if not daily - so rapid, efficient measurements were needed to justify the effort and expense of prototyping and maturing the design. Although not necessary for a first-generation PSA variant, the possibility of including a mechanical rejection system capable of ejecting sub par specimens from the main lot was discussed as a future direction that might be pursued.

Prior to initiating an independent development effort, existing commercially-available systems were investigated as possible solutions. Several companies specialize in this area, and the hope was that while an existing system may not prove usable out of the box, modifications to either the mechanics or the internal software might produce a successful result. Unfortunately, the focus of commercial particle analysis resides within size ranges where airborne or liquid suspension is of interest - namely smaller than $5 \mu \mathrm{m}$. Within this size regime, systems are designed for particle size sampling as opposed to counting. Furthermore, those systems that do advertise counting capabilities use light scattering rather than light blocking to provide their analysis and cannot produce any shape data. Such a system was tested by the program and failed to show any promise. Vendors do offer means of more thorough analysis using techniques similar to the backlit optical techniques employed within the AGR program. While offering a more complete size and shape analysis, these systems provide no means for counting and do not provide the resolution needed to use such a system to meet typical coated particle fuel specifications for size and shape.

Given the unfeasibility of adapting existing optical systems, it became clear that an independent approach to counting and sizing particles was necessary. Literature searches and investigation into previous particle fuel development efforts revealed that a similar problem had been encountered decades earlier by Austrian researchers in support of a German foray into high-temperature coated fuels. ${ }^{3}$ The basic concept was to pass particles across a light source, either by gravity alone or driven by an external fluid power source such as compressed air to push the particles through the system or a vacuum pump to provide suction force. As the particle passed into, through, and out of the light's path, a photodetector would be positioned in the 'shadow' created and pass data through a digital acquisition (DAQ) to be analyzed using a piece of software written specifically for this purpose. Beginning with a baseline light level detected when no particle is in the field of light transmission, the light recorded by the photodetector will drop to a minimum level as the full particle eclipses the beam before returning to baseline as it exits the system. A general schematic of this is shown by Figure 2 and Figure 3. 


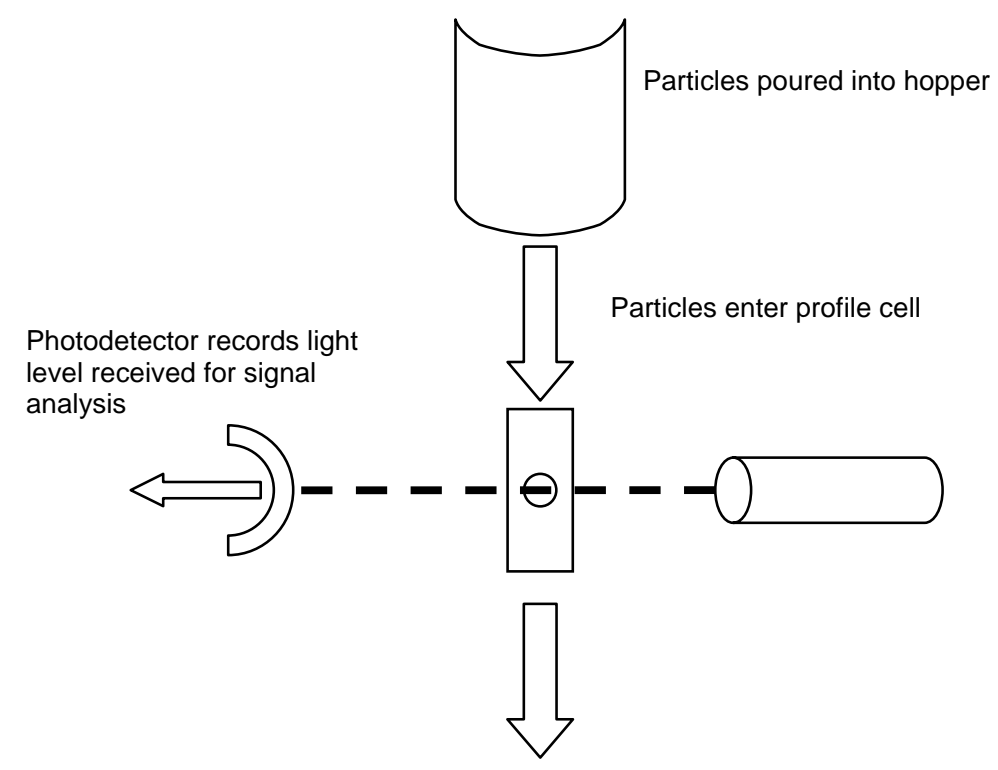

Figure 2. General concept of PSA using light beam obscuration.
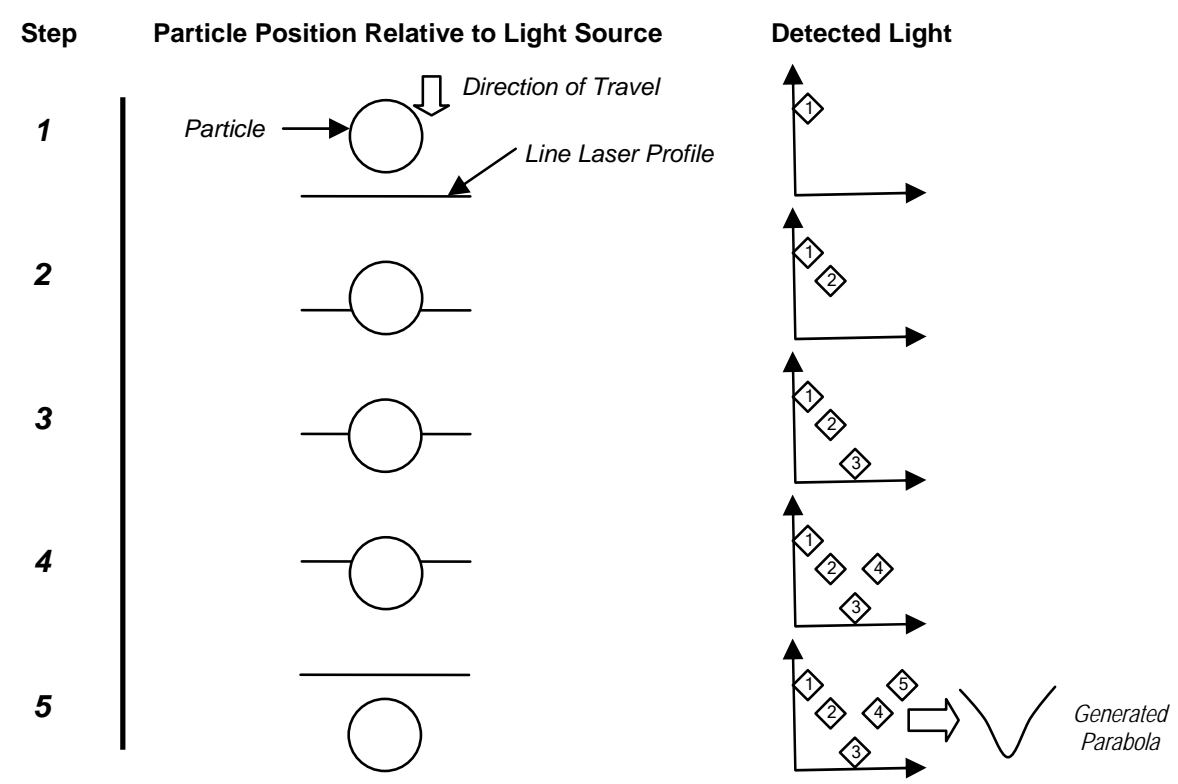

Figure 3. Schematic of particle falling through light beam and generated parabola.

The paper presented by Wallisch and Koss claims several impressive attributes of their PSA development effort. They found that a sample's size distribution, cited to within a few microns, could be calculated for particles ranging from 100 to $1500 \mu \mathrm{m}$ in diameter at a rate of 100 particles per second. However, the main difference between their design and one required for integration into the AGR program was the lack of shape data for an entire sample. While a separate shape factor using a multi-pass particle transport option is included in their design, it is performed only on a few individual particles extracted from the sample and takes much longer than the main process. This information (if performed on a sufficient fraction of the sample to ensure statistical relevancy) may allow 
conclusions on the aspect profile distribution within the sample, but does nothing to affix shape data to a particular event. As such, it appears that no attempts were made to verify what diameter their PSA output when a particle became less spherical or match a recorded event's diameter to an aspect ratio or other shape factor quality indicator.

Regardless of the particular differences in measurement and analysis methods, the Wallisch and Koss PSA provided critical guidance in the early phases of the design process. Four main design components can be identified from the concept outlined. First, a method of transporting particles from an input location to an output is necessary. Second, a funneling of the particles must occur to direct them through the light source in a single stream. Critical to the success of the system is a means of providing a light beam/detector pairing with characteristics such that the system can record small changes in particle size. Finally, the software package and imbedded algorithm used to count and size each particle will perform the analysis asked of the PSA.

\section{Particle Transport}

Other phases of the AGR characterization program provided extensive experience with design and implementation of coated particle handling systems using gravity alone to move a batch of particles from one location to another. However, two main aspects of the PSA design dissuaded use of gravity alone as the driving force behind particle transport. First, existing gravity feed systems within the AGR program were forced to implement vibrating surfaces to prevent particles sticking together or clogging inlets surfaces. Since the PSA system required a light beam and detection system that would rely on constant comparisons between a baseline and real-time data, adding structural perturbations would only complicate data noise within the process. Additionally, the prospect of implementing a particle sorting or rejection system to the imaging and counting capabilities suggested that an active transport medium which might be adjusted or diverted to aid in this rejection would be preferred to a static gravitational force.

A vacuum system was identified as the method of choice given its ability to connect with a cyclone separator to remove particles from the generated velocity stream. Integration of particle transport with the medium driver at a location downstream from the particle inlet also allowed for a straightforward hopper-style particle staging method. If compressed air was used to aid in transport, some means of 'injecting' the particles into the flow region would be needed and could prove difficult. Furthermore, cyclone separators are commonplace in the particle handing industry and represent a fairly well-understood technology. A suitably sized pump is connected to the top of a cylinder coupled with a conical section at its base. Particles are fed into the side of the unit and circle in the cyclone's velocity vortexes induced by the negative pressure at the top of the separator, as shown in Figure 4. The system is sized such that the particles of interest are too heavy to be lifted into the vacuum line, instead falling into a collection chamber at the base. The cyclone separator designed for the PSA was designed using all type-304 stainless steel. The main body tapers down to meet a smaller flange which is welded to the main body. The flange contains several holes which allow for the connection of a vessel for 
collection of particles exiting the system. A removable top section containing the vacuum connection attaches to the main body via six machine screws fed through a neoprene gasket threaded into the main body. Access to the separator body is necessary for system cleaning.

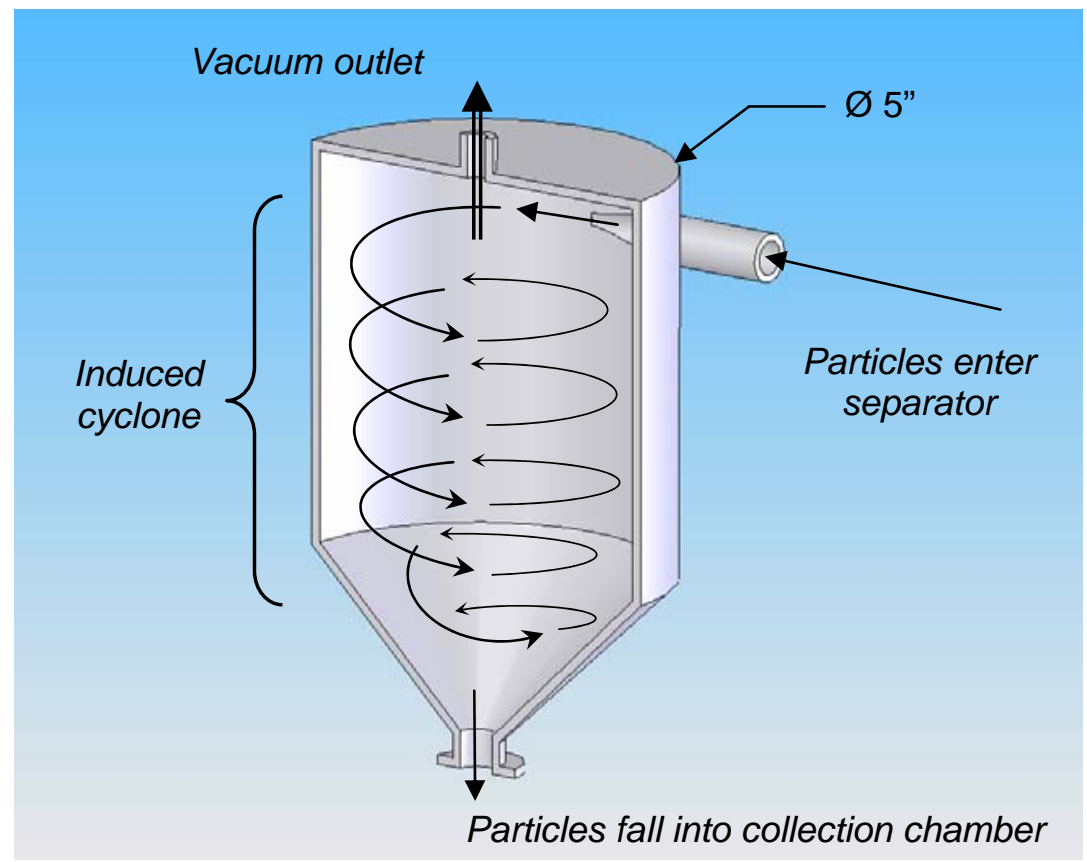

Figure 4. Diagram of key features of cyclone separator.

With the pump providing a vacuum to assist in transport of the particles through the system, a means of delivering particles to the imaging cell required considerations only to ensure that particles were not able to lodge themselves at any point within their path. Meeting this criteria meant designing the PSA particle transport system without any obstructions that could restrict particle motion. As the anticipated particle feed rates were in excess of 30 throughputs per second, even a momentary stoppage of motion could result in a backup of the entire system. The tubing transport system was designed in a telescoping fashion to meet this goal. As a particle traverses the system, the diameters of each tube length and component increase to avoid providing ledges that could trap or reflect particles. A schematic of this geometry is shown in Figure 5. The added benefit of an increasing cross sectional area as the system progresses from the inlet to the cyclone separator is that the volumetric flow rate induced by the vacuum will also increase due to the reduced wall resistance. This will help to minimize the total time spent by each particle inside the system and aid in increasing total processing rates. 


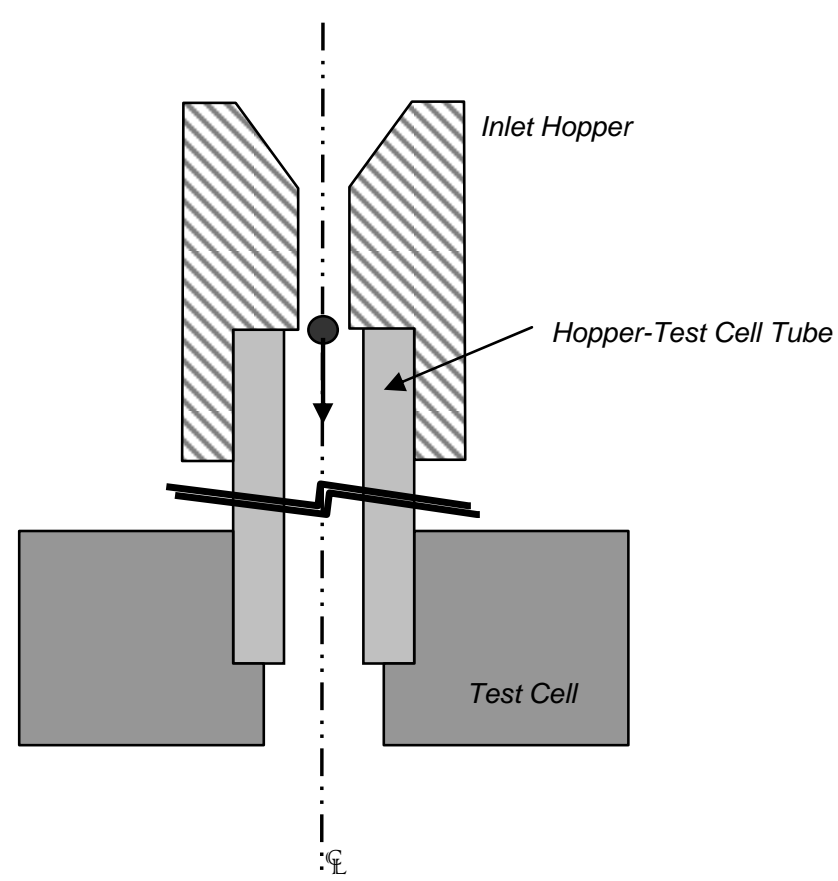

Figure 5. Cross section of particle path between hopper and test cell.

A traditional hopper geometry was designed to connect directly to a $15 \mathrm{~cm}$ length of 316 stainless steel transport tubing, which in turn would deliver particles to the test cell for measurement. The hopper was machined of 6061 aluminum containing an interference fit where the hopper-test cell tube joined it at the bottom. Existing data provided by optical shadow analysis was used to determine the minimum permissible openings for a particle lot of a given mean diameter to ensure the distribution in size and shape would not result in outlying large particles becoming trapped within the system.

Since the PSA design was originally specified to include particle sizes ranging from 350 $\mu \mathrm{m}$ diameter UCO kernels all the way up to $1300 \mu \mathrm{m}$ diameter TRISO particles overcoated with a carbon matrix in preparation for compacting, it was understood that several different size test apertures would be required to accommodate this wide range in diameters. During the initial stages of development, ORNL health physics personnel determined that sending uncoated kernels through the system posed a high risk of generating airborne uranium dust. This precluded any attempts at using the PSA on these smallest diameter particles. Instead, the decision was made to pursue two main particles: the $820 \mu \mathrm{m}$ full TRISO and the $1300 \mu \mathrm{m}$ overcoated particles. Two test cells were designed to provide the ideal clearance for particles of each type. The optimal aperture cell width was calculated with regard to two primary problems that had plagued the early experiments: the need to prevent more than one particle from entering the test cell at a time, and the tendency of particles to become lodged within the laser sight opening.

Typical TRISO particle lots had mean diameters of roughly $800 \mu \mathrm{m}$ with standard deviations of $10-15 \mu \mathrm{m}$. A minimum system diameter of $120 \%$ of the expected mean particle diameter was found to be a sufficient tolerance based on observed particle size distributions to ensure compatibility with all conceivable TRISO variants. The upper 
limit was set to prevent two particles seizing within transit by becoming wedged against each other. This parameter was considered to be $180 \%$ of the expected mean particle diameter. To achieve the required telescoping of diameters from the hopper to the test cell exit, four dimensions were used for TRISO particles. The hopper feed was machined to $1.02 \pm 0.5 \mathrm{~mm}$, followed by a $1.12 \pm 0.01 \mathrm{~mm}$ ID section of tubing constructing the hopper-test cell tube. This tube then connected to the TRISO test cell that had a bore diameter of $1.24 \pm 0.03 \mathrm{~mm}$. Finally, the test cell exit tube ID was $1.42 \pm 0.01 \mathrm{~mm}$. The test cell exit tube emptied into a $25 \mathrm{~cm}$ length of $0.635 \mathrm{~cm}$ ID braided PVC tubing that led to the cyclone separator. At this point of transition from test cell exit tube to the cyclone separator, because there was no longer a need to maintain a single particle stream and in order to reduce resistance in the particle transport plumbing, the system was widened to remain much larger than all TRISO particles at each coating state.

\section{Light Obscuration}

Passing a particle across a light source and measuring the resulting change in light detected by a photoreceiver behind the particle is the critical component of the PSA design. The first step in this process is designing a light beam generation and detection system sensitive and stable enough to detect the presence and size of the TRISO fuel particles with high resolution and reliability. For light generation, a 15-degree fan angle laser was chosen operating at a wavelength of $650 \mathrm{~nm}$. The laser was provided by StockerYale, specifically a Micro-Focus (MFL-650S-10-15-185-SD) designed to produce a beam $35 \mu \mathrm{m}$ thick at a focal distance of $185 \mathrm{~mm}$. This laser was matched to a suitablysized power supply. A basic geometric representation of the laser projection through the target cell is shown in Figure 6.

The photoreceiver selected for the design was the New Focus 2032 large-area model. This specific receiver has three gain settings and is responsive to wavelengths between 190 and $1100 \mathrm{~nm}$. The three distinct gain settings (2e3, 1e5, and 2e6 V/A) provided some flexibility with the output signal range in mating with signal processing hardware. Additionally, the $5.8 \mathrm{~mm}$ detector window diameter allowed for centerline alignments accurate only on the order of $1 \mathrm{~mm}$ rather than the much tighter needs a smaller window would require. The middle gain setting was chosen for PSA operation for two reasons. Most importantly, this produced a signal that best matched the input range of the analogto-digital converter (ADC). In addition, the noise characteristics of the mid-range setting is also superior to that of the low-range gain by an order of magnitude. Since the PSA will investigate changes in particle sizes on the order of $1 \mu \mathrm{m}$, maintaining low uncertainties throughout the process is crucial. To complete the obscuration subsystem, a neutral density filter was installed on the front of the photoreceiver with an optical density of 0.6. 


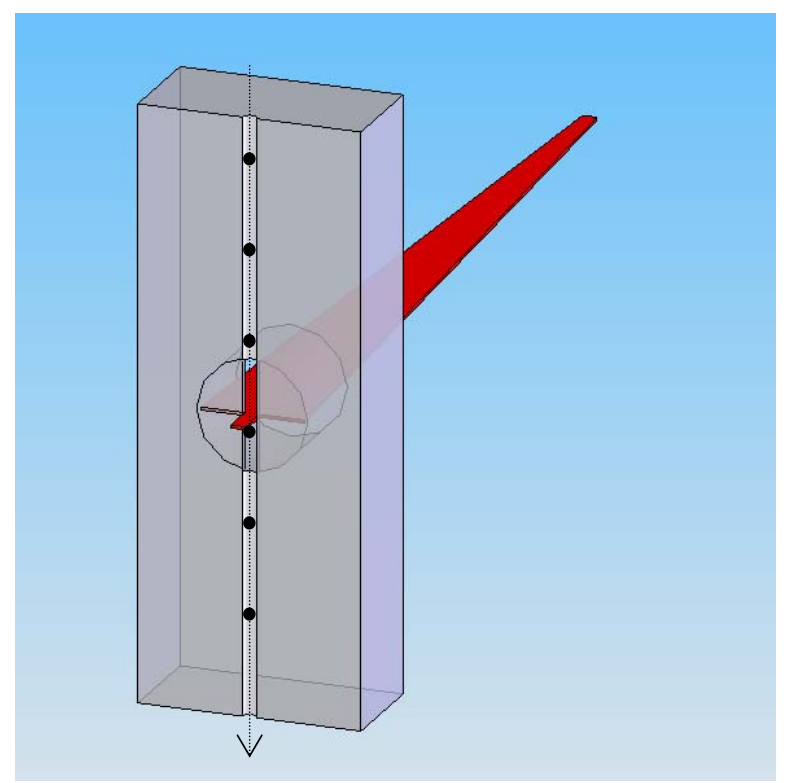

Figure 6. Cutaway view of cell showing particle's path through line laser.

Even though the fan angle of this laser was relatively benign, a focus point nearly $20 \mathrm{~cm}$ away from the mounting position proved to cause some distortion when particles passed along the edge of the test cell. The angled beam resulted in a portion of the target area being shielded from the detector. Some fraction of a particle passing through the cell at the extreme edge could pass through this dead zone and result in an under-representation of the particle size in the obscured light signal. This phenomenon is shown in Figure 7. This condition would not greatly matter if particle counting alone was of interest, but when sizing is also considered it requires attention. To address this, a $100 \mathrm{~mm}$ cylindrical lens was added to the system on an adjustable mount to collimate the laser. A series of calibrations were performed to determine the correct positioning with respect to the laser and detector positions to ensure that the original laser profile was not distorted with the addition of the lens. 


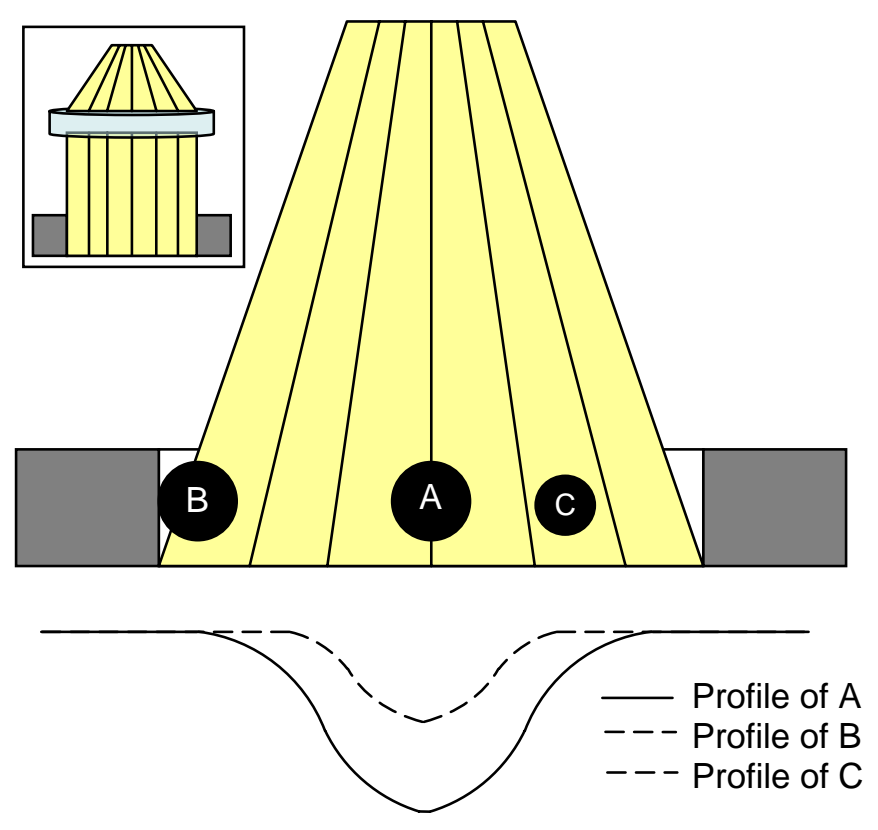

Figure 7. Particle imaging problem caused by laser fan angle. Profiles for similarly-sized particles are shown with exaggerated geometry demonstrating the under-representation of particle size that can occur without a beam collimating lens. Insert shows addition of lens.

\section{Data Analysis}

In order to achieve the stated PSA design goals, the signal produced by the photoreceiver as the particles pass through the laser's path must be successfully translated to size and shape data. From the initial phases of the design, it was assumed that a piece of analysis code could be used to perform this task. The photoreceiver ADC is handled via National Instruments 6111E data acquisition card which in turn passes the signal to a PC. Analysis of this signal is performed using a suite of customized algorithms that will be detailed in the 'Software' section.

Numerous beam calibration methods were attempted in an effort to achieve the best particle count and size results. Early in the development process, it was discovered that the beam profile was not uniform across the photoreceiver. If the laser intensity is not consistent across the width of the detector, the fraction of light obstructed by a particle falling through the test cell will become a function of the particle's horizontal location within the cell and thereby reduce the accuracy of the sizing algorithm. This phenomenon is shown in Figure 8. 


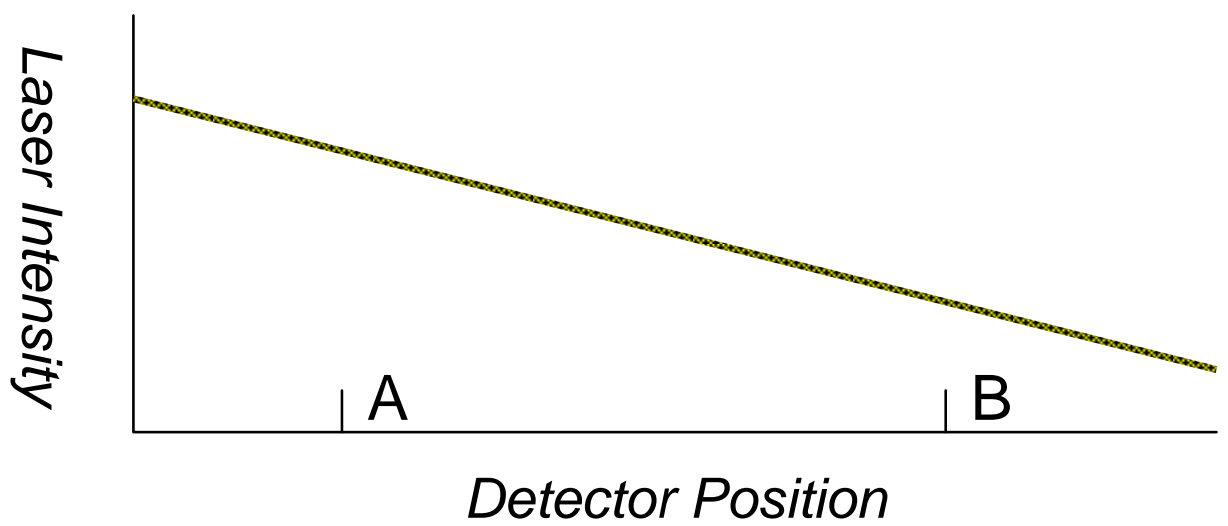

Figure 8. Laser intensity across photoreceiver. A particle passing in zone A will obstruct a larger fraction of the total light detected compared to a similarly-sized particle passing through zone $B$.

Fortunately, these inconsistencies in intensity do not exist throughout the entire width of the collimated beam and if the beam trajectory is adjusted it is possible to locate a region where the variations are nearly zero. This is achieved through the use of a speciallydesigned vertical slit aperture that can be used with a calibration portion of the computer code. For a given laser orientation, the slit is moved incrementally along the horizontal axis to sample the intensity of the transmitted light as a function of the horizontal position. As the slit is moved, the stored readings accumulate to build a mean and standard deviation for that laser orientation. Should the standard deviation exceed a prescribed value, the laser orientation is changed slightly and the process repeated. A graphical display is available to aid in diagnosing the horizontal profile. For example, a profile such as that shown in Figure 9 would suggest moving the laser such that more of the rightmost region in the profile would pass through the detection zone.

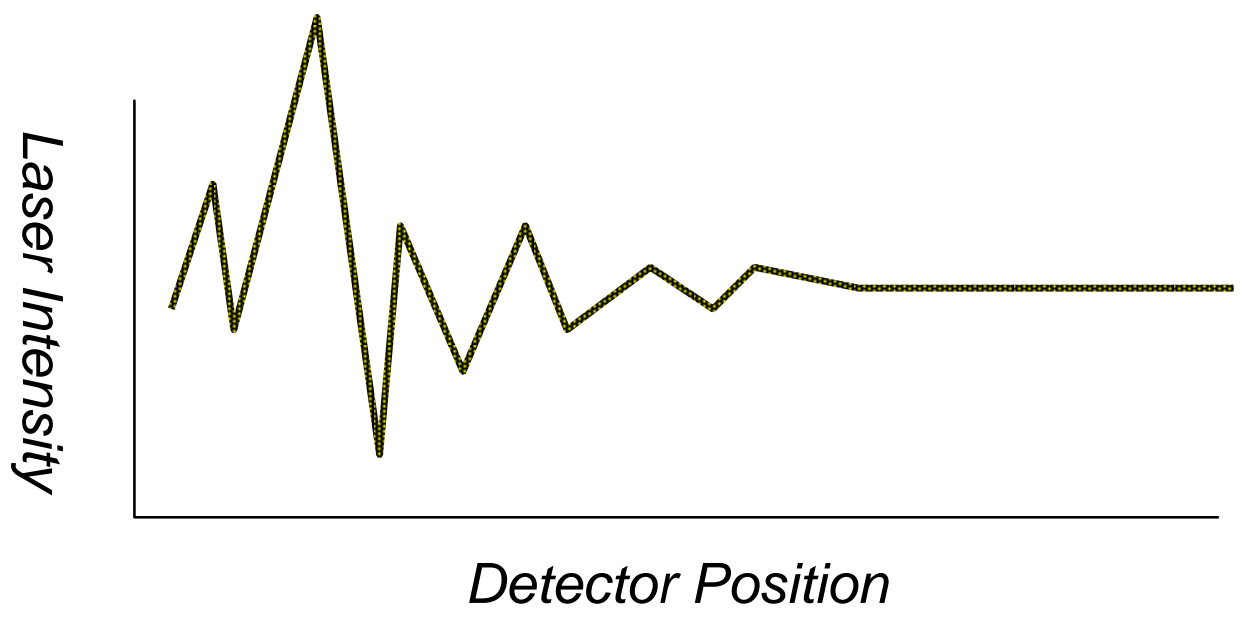

Figure 9. A 'bad' laser profile that would degrade the quality of particle shape measurements. Given that the flatter region appears on the right-hand side of the profile, shifting the laser focus left to center this region on the test cell would lead to better results. 
The source of this inconsistency was never successfully verified. Each time the laser was powered up, intensity variation was observed in different regions of the laser beam at differing severities. Non-uniformity and noise generation within the line laser profile remained a consistent concern throughout development, as uncontrollable variations within the light source will reduce the accuracy and reproducibility of PSA measurement. For further improvement of the system, consideration should be given to replacing the current laser with a more stable laser or perhaps an alternate light source.

With a region of minimal intensity variation identified, the next calibration step to be completed is the measurement of the baseline intensity value. This measurement is critical to the success of both particle counting and sizing attempts, as an accurate comparison of any obstructed light level relies on an accurate unobstructed quantity. This again is achieved with a simple operation defined within the code's user interface to 'snap' a measurement of the current photoreceiver state and use this value as the baseline. Typically, the user performs this calibration a few times in succession while noting the value recorded each time as a check to verify the stability of the signal and the integrity of both the beam and photoreceiver mechanical mounts and all connections within the system. Internally, the code samples the average of a 50,000 point buffer ( 0.01 seconds) to identify the baseline.

Finally, the last calibration required involves a calibration run performed by sending a known number of spheres through the system with a known diameter. Stainless steel spheres with known diameters to within a few microns are available from numerous sources and such a sample of $800 \mu \mathrm{m}$ spheres were used for this calibration. The purpose of this calibration run serves only to match internal code parameters to those of the current particle run. Specifically, the scaling factors required for a TRISO run where particles are approximately $800 \mu \mathrm{m}$ and an overcoated particle run where the average particle is $1200 \mu \mathrm{m}$ in diameter will require unique values.

\section{Software}

The final portion of analysis is conducted by the software code written specifically for the PSA in $\mathrm{C}++$ and Visual Basic programming languages. As data is fed into the computer, the software package must be capable of identifying when a particle has passed through the line laser, extract size and shape data from the event, and then allow all data to be sorted and analyzed following the completion of a PSA run on an entire sample. To facilitate use of the system by an operator untrained and unfamiliar with editing code, a simplified user interface is required. Finally, the system must be robust enough to ensure that all conditions of operation are foreseen and unexpected events do not paralyze the process.

Determining the particle size from a light obscuration profile was the subject of initial PSA concept exploration. The mathematical model for a band of light obstructed by a particle passing through it are discussed in an earlier work. ${ }^{4}$ The method of choice identified by the authors for implementation into the PSA software was based upon the 
finding that the diameter of a particle is proportional to the minimum of the particle's obscuration profile. The proportionality constant will depend on a number of factors such as test cell geometry and light conditions. Since generation of an explicit form for the proportionality constant would have required substantial effort outside of the focus of PSA development, a calibration run was designed as discussed in the previous section. The purpose of the calibration run is to match the experimental conditions at the time of a PSA run to a proportionality constant. This is achieved by running a small number of spheres calibrated to a known diameter through the system thereby associating the calculated obscuration profile with a specified diameter.

Following the calibration, the PSA run commences. As particles pass through the line laser, the software identifies each event each time the detected light level drops below a predetermined fraction of the unobstructed value. The software then stores a diameter as well as a fit quality to each event. The fit quality is a rough comparison of the event profile with that of the profile generated by a perfect sphere. This value has three uses: identifying false events, identifying a departure within the system from standard operating conditions, and potentially calculating aspect ratios. Following the completion of the run, a histogram is exported in .csv format for viewing. Software was also written to aid in viewing and sorting the event profiles. Figure $\mathbf{1 0}$ shows a screenshot of the interface written for PSA operation.

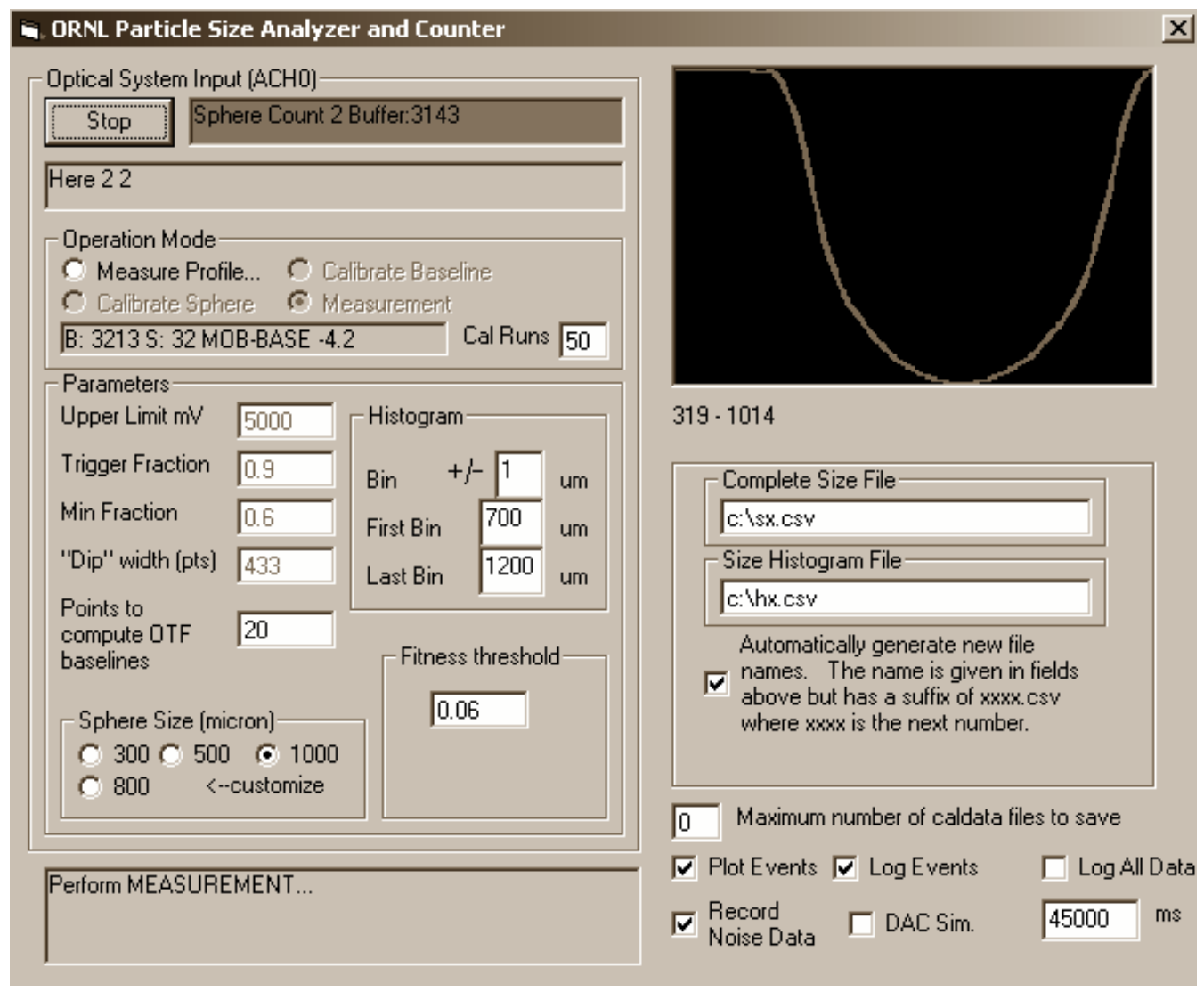

Figure 10. Screenshot of PSA graphical user interface showing a typical event and analysis settings. 


\section{PSA Operation and Development}

As is expected with any mechanical process engineering effort, substantial refinement was required after the design of each system component had been completed. An aluminum housing was constructed to provide a fixed mount for the laser, test cell, and photoreceiver. In addition, panels on the sides and above the light producing and detecting equipment prevented exterior light sources from interfering with the process. Figure 11 and Figure 12 show the support structure and enclosure in place, as well as the internal components of the PSA.

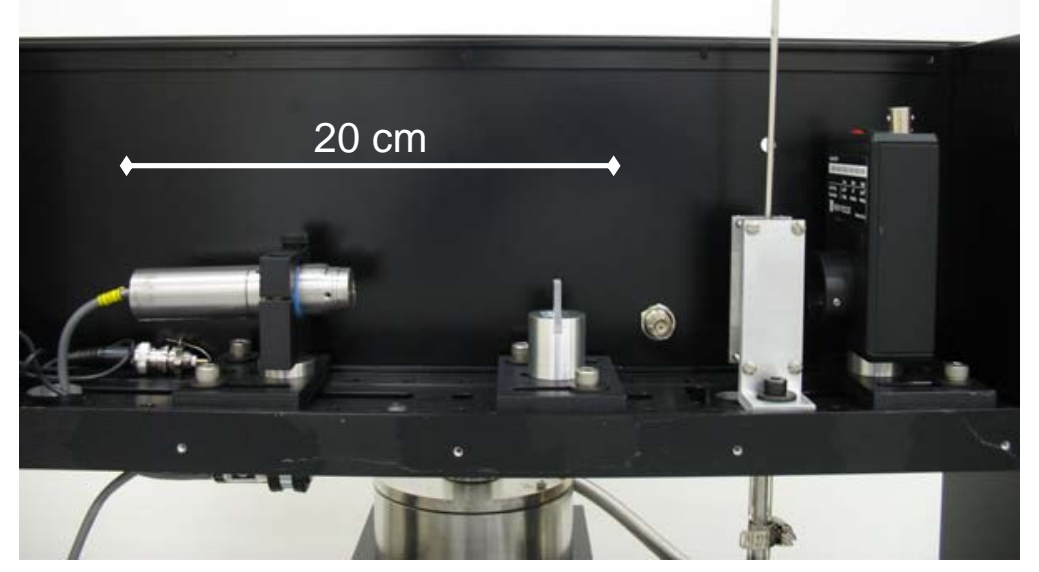

Figure 11. PSA with side and top panels removed showing (from left to right) laser, collimating lens, test cell, and photoreceiver.

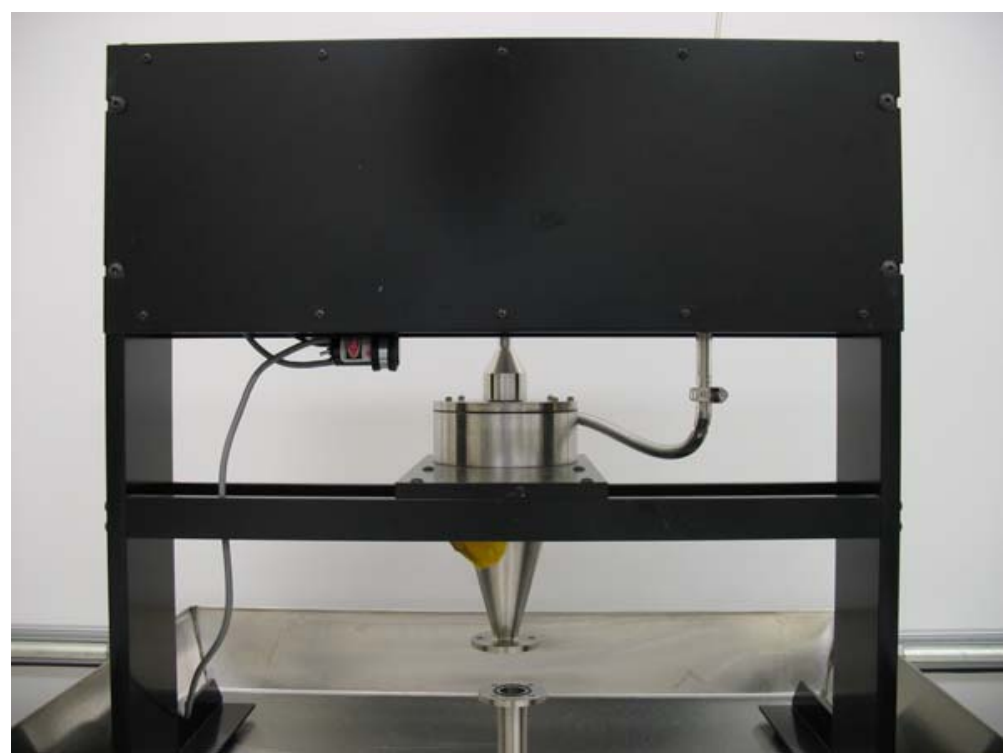

Figure 12. PSA as seen prior to operation with all panels in place. Cyclone separator is visible below enclosure.

The following sections highlight the most relevant issues encountered in the PSA development process. In addition, the specific capabilities of the PSA are discussed along with areas where further improvement may be possible. 


\section{Particle Transport Refinement and Manual Delivery}

During initial testing and software troubleshooting phases of the design, the means that particles would be delivered to the target cell were unknown. It was generally anticipated that a hopper feed system would be utilized, but given that the cell design itself was undergoing constant modification and redesign, the delivery mechanism design was delayed. In this time period, particles still needed to be transported from the laboratory weigh pans to the cell inlet tube. Since a vacuum was being pulled on the system via the cyclone separator, it was natural to use this suction to pull particles into the inlet tube. This was accomplished by attaching plastic tubing to the inlet tube and then drawing particles through the target cell directly from the weigh pan.

This crude technique allowed development of the software analysis and target cell to proceed to completion. While particle delivery undoubtedly plays a role in the overall performance and accuracy of the system, attempting to refine initial steps in the procedure in the presence of errors and problems further downstream is counterproductive and prevents efficient identification of error sources.

With the final test cell design and inlet tube dimensions determined, large quantities of particles were ran through the system using only the inlet tube suction method. Numerous tube materials, diameters, and lengths were tested. The most critical parameter was the tubing inner diameter, as would be expected. In order to maximize the flow through the tube, tube diameter should be pushed as large as possible before excessive particle clogging becomes an issue. Experience revealed that this particle clogging limit would be reached before suction tube fitting to the cell inlet tube would become a problem.

Given that the main purpose of the tubing suction system was to facilitate particle transport into the test cell, the minimum length of tubing required was set by the specific operator's comfort level. If the operator was able to hold the particle tray in one hand and use the other to maneuver the suction tube, the tube would only need to be approximately 4 inches long. However, given how easy it is to spill particles out of even large weigh trays and the time that particle suction takes, it is unreasonable to expect tray suspension to be possible. This led to what became the standard technique of placing the weigh tray on the top of the laser enclosure and providing particle suction using a slightly-longer tube section on the order of 6-8 inches. Increasing the tube length longer than this proved difficult for multiple reasons. First, the flow rate delivered by the vacuum at the suction tube inlet was insufficient to pull particles in when the tube length exceeded roughly twenty inches. This was not long enough to reach the table top from the target cell inlet. Furthermore, excess length only introduced additional head losses and tubing bends that made particle clogs more of a problem.

Even with the limited length, particle clogs were still a problem with the hand suction feed system. While tubing bends were sometimes points of congestion, the most frequent clog point occurred at the entrance of the test cell inlet tube. The reason for this was the 
method uses to attach the suction tube to the inlet tube. Inlet tube sizing was calculated carefully to preclude two particles traveling into the tube simultaneously. As such, the plastic tubing had to be forced over the outside of the tube to form a tight seal. This is shown in Figure 13.

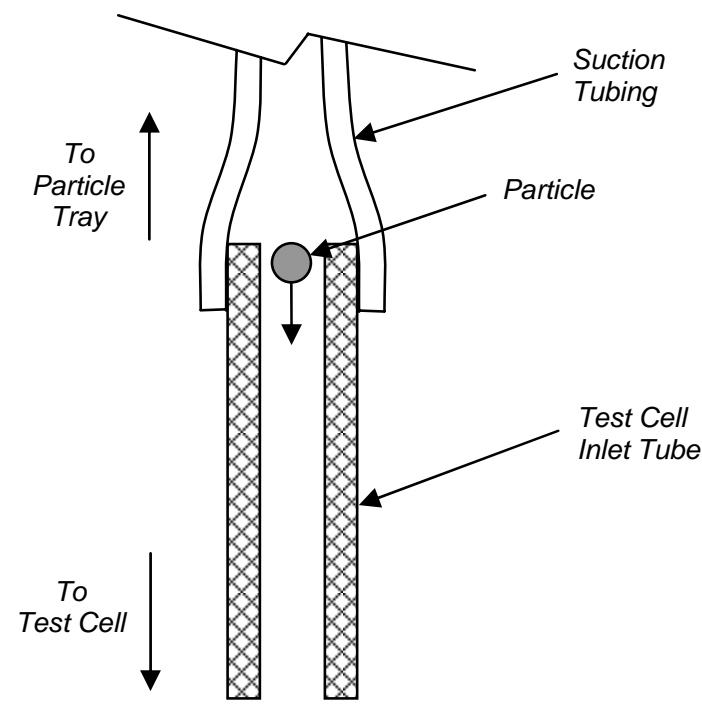

Figure 13. Suction tube attachment to target cell inlet tube.

The benefit of using such a simple connection between the two tubes was ease of installation and removal. During the development stages of the design, the target cell was frequently removed from the laser enclosure for inspection and modification. No mechanical clamping system was used since the vacuum served to pull the suction tube even tighter onto the inlet tube. Experimentation during usage determined that $1 \mathrm{~cm}$ overlap of the suction tube onto the target cell inlet tube was sufficient to prevent the suction tube from accidentally detaching from the inlet during particle intake. The one obvious drawback to this technique was the creation of a ledge on the inlet tube where particles could catch and become clogged. This problem is shown by Figure 14. As the particle flow rate is increased, it is possible for multiple particles to enter the expanded throat of the suction tube simultaneously. With the exposed lip providing a surface to slow the particle and obstruct its path, particles can become pinned against each other. During the suction process, typical flow rates are 10-20 particles per second, quickly escalating the small clog to an entire blockage of the suction tube throat before the operator is aware of the clog. Typically, two indications of a clog would be quickly apparent: the PSA user interface would stop showing particle counts despite the fact that particles were still being fed into the suction tube, and the clog would greatly decrease the air flow into the system causing the vacuum pump to operate at a higher pitch. 


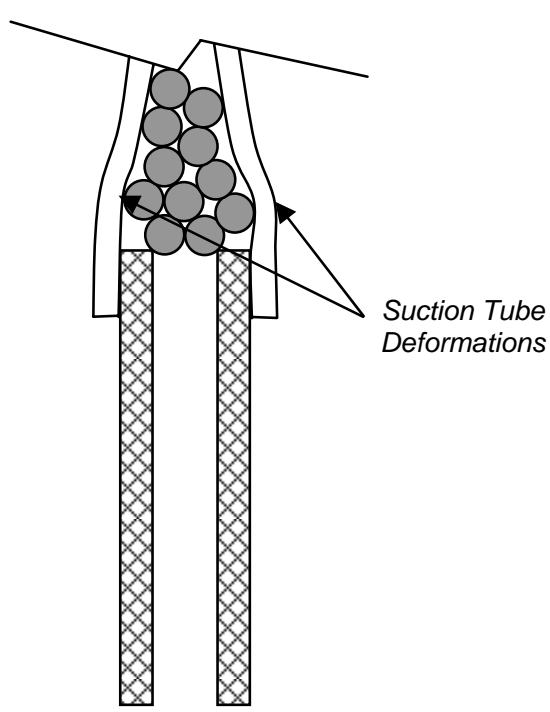

Figure 14. Particle clog caused by exposed inlet tube edges and suction tube deformation.

Fortunately, freeing the clog was a fairly straightforward process provided the suction tube material is sufficiently flexible. Simply pulling and rotating the suction tube throat allowed the stuck particles to shift until those on the bottom which initially created the stoppage were moved off the inlet tube lip and passed into the target cell. The flexibility of the suction tube does allow more particles to collect into the throat region as the tube is able to expand outward. The alternative to this is a suction tube that is more rigid.

Experimentation with this did not result in less particle clogs. Conversely, it made freeing the clogged particles more difficult because the rigid suction tube cannot be manipulated to allow the caught particles' exposure to the freestream vacuum of the inlet tube.

The regrettable side effect of this technique is unavoidable damage to softer coatings. The TRISO particles analyzed throughout the bulk of the development had surface coatings tough enough to withstand the impacts suffered during attempts to free a clog. However, the final layer of graphite overcoating was found to be too soft for this method. Not only would particles push against the other particles in the clog, but the lip of the inlet tube provided a considerable surface for partial impacts. The few samples of overcoated particles that were run through the PSA using this delivery method produced only a few intact particles in the collection chamber at the bottom of the vacuum separator; most particles were stripped of their overcoating and reduced to TRISO particles along with chips of overcoating and graphite dust.

\section{Damage to Overcoated Particles}

Several attempts were made to characterize overcoated particles using the PSA. While the size and shape of the overcoated particles is less critical since they are compacted within a graphite matrix to form the fuel sticks, accurate data on the exact number of particles within a lot as well as size information to determine overcoating thickness 
remains valuable. In addition to the size differences between a TRISO and overcoated particle, the carbon used in overcoating is much softer and less-adherent than the OPyC coating found on the outer surface of a TRISO particle. This difference caused significant problems when PSA runs were attempted, as the overcoating broke apart during the particle's transit through the system. When the vacuum was turned off and the particles retrieved, only a small fraction of those sent through possessed intact overcoatings. Clearly a destructive characterization technique is undesirable, and PSA use of overcoated particles was discontinued.

A PSA for overcoated particles could be developed, but the transfer medium would likely have to rely on a means other than a vacuum draw. The larger overall size of an overcoated particle may lend itself to employing a gravity feed, such that particle impacts on the walls of the transit tubing would be greatly reduced. Furthermore, since size information is of less interest, focusing overcoated analysis on counting alone would require a much less controlled detection system.

\section{Baseline Degradation}

Once the switch was made from stainless steel spheres and $\mathrm{ZrO}_{2}$ surrogate particles to TRISO fuel, the carbon coatings introduced additional concerns. After only a few runs using the TRISO fuel, a fine graphite coating was observed both inside the test cell window and inside all clear plastic tubing used throughout the system. The presence of carbon alone is not necessarily a problem, as much of the characterization equipment used in the AGR program must be continually cleaned. However, successive TRISO runs saw a continual degradation in the baseline light levels detected by the photoreceiver. This was observed to be caused by an accumulation of a thin carbon film on the test cell window. Recalibrating the baseline before each run is simple and takes only a few seconds, but if the baseline deteriorates after each run it can safely be assumed that the baseline level is continually deteriorating during each run. This indeed proved to be a significant problem, since events are triggered by a departure of the light level from the baseline. As the baseline level falls off from the stored value, the likelihood of false positives increases. This fear was verified as particles were continually fed through the PSA beginning with a clean system. After approximately 10,000 particles, a few events were stored that were in fact only small noise variations within the system. These counts are easy enough to pick out due to their poor fit quality, but a poor baseline reference reduces the quality of all sizing attempts within the run.

The solution to this issue was discovered during attempts to merely quantify the problem. Beginning with a clean system, a sample lot of roughly a thousand particles was run through the PSA again and again, with a baseline value recorded before each pass. It was discovered that the baseline level drops at a fairly high rate initially, but then stabilizes to a constant level. This is assumed to be due to the fact that the cell window can pick up a certain film thickness of graphite and then reaches equilibrium. From this information it was determined that all future runs following system disassembly and cleaning should 
only be performed following sending roughly 10,000 particles through the system to build up an equilibrium graphite coating on all surfaces.

TRISO particles tend to be sooty on the surface when removed from the coater. When particles are poured into a stainless steel tray and moved around, a thin layer of carbon residue builds up in the tray, similar to what has been observed in the PSA. A procedure for washing particles prior to compacting was developed to remove this carbon soot as well as other possible surface contaminants. It is likely that the carbon contamination in the PSA was due to this loose soot as opposed to abrasion and removal of the hard outer pyrocarbon. Future development of the PSA should consider the advantage of washing the TRISO particles prior to analysis.

\section{Laser Profile and System Noise}

Once the system was robust enough to begin actual experimentation based upon running complete particle samples through the PSA and comparing the data generated, the question of the laser integrity arose once again. The variations within the line laser profile itself had been observed from its original coupling with the photoreceiver. Adjustment of the line laser's position across the test cell undoubtedly reduced potential errors, but it was unknown how much this profile varied during the particle run.

This issue has been only partially explored to date. Complete streams of photoreceiver output data have been examined and these values remain largely constant, especially when compared to the values during an event. Second, a single $800 \mu \mathrm{m}$ steel sphere was sent through the PSA many times in succession, resulting in particle diameter agreement within $4 \mu \mathrm{m}$. Calibration data from the steel sphere supplier reports that diameters are constant to within $2 \mu \mathrm{m}$, equating to aspect ratios below 1.005. This shape imperfection, although minuscule, may introduce additional inconsistencies in the measurement and will be discussed later in more detail. A resolution of $2 \mu \mathrm{m}$ is approximately the limit of current shadowgraph imaging capabilities, so the steel particle would be classified as spherical within the range of TRISO particles. Given this assumption, the $4 \mu \mathrm{m}$ variation is most likely due to inconsistencies within the line laser or noise elsewhere in the system. For full verification of both line laser consistency and possible noise within the laser-photoreceiver pairing, a more complete barrage of testing is needed.

\section{Particle Bounce Back}

The key aspect of using a light obscuration technique to count and size particles lies in maintaining process control such that the data provided for analysis can be confidently linked to a recognized event. In the case of the PSA, this corresponds to light data recorded in the absence of a particle, followed by a parabolic profile generated as one particle passes into and then out of the line laser, and then finally an unobstructed profile. Analysis of data gathered when this chronology is broken or unknown will result in large errors. For example, a fundamental assumption of PSA operation relies upon each 
particle being measured only once. Early investigation into the size and geometry of test cells revealed that, despite a strong vacuum drawn on the system outlet, occasionally particles would somehow oscillate within the test cell and trigger not one, but three events (original event, second event as particle passes upward through beam, and third event as particle passes again downward through cell window).

This behavior was discovered while trials were being performed with only one particle sent through the PSA and three events were recorded rather than just one. With the installation of size-specific test cells and transport tubes containing much tighter particle clearances, this behavior was no longer observed. Furthermore, the excellent count reproduction exhibited by the PSA suggests that no particle transport anomalies exist within the current configuration.

\section{Double Peaks}

One of the earliest issues observed during PSA operation were 'double peaks' recorded by the photoreceiver when two particles pass through the test cell in close proximity such that the detected light level does not return to baseline between the particles. Since the software was instructed to end an event when the light level returned to the baseline level, the particle pair is recorded as a single event. This results in only one particle being counted and a complete failure in the sizing algorithm. Even though extensive effort was expended in the design of the test cells for each target diameter to ensure that the cell diameter was only large enough for one particle to pass through at a time, a slight offsetting of two particles stacked in rapid succession can still generate a false profile. A schematic of this phenomenon is shown in Figure 15. 

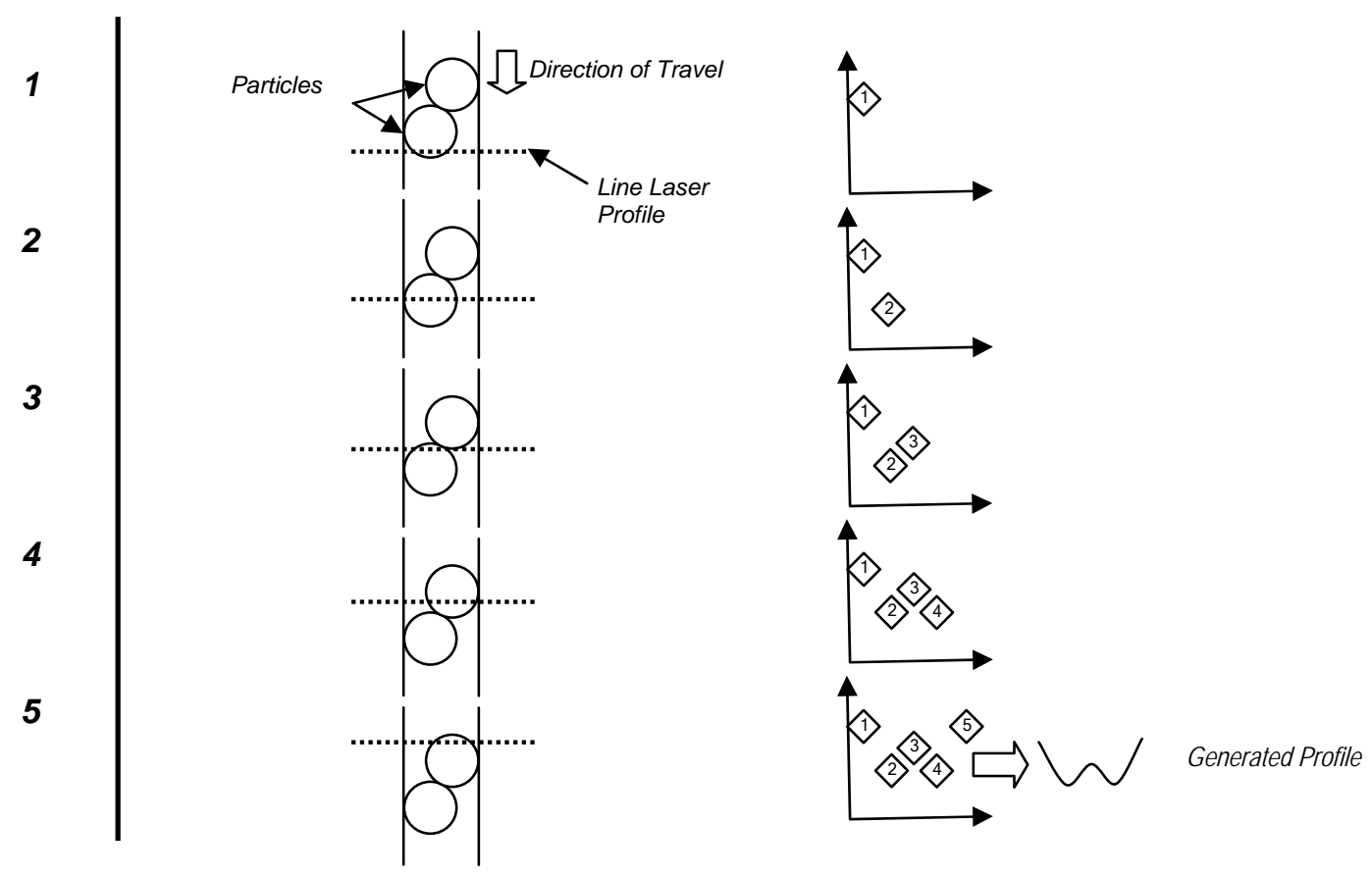

Figure 15. Schematic of particle pair falling through line laser beam and generating a double peak.

This event occurs with relatively low frequency. Less than $0.1 \%$ of all recorded events are double peaks, and this is easily corrected for when counting particles alone is of interest. During exploratory efforts attempting to isolate causes for the problem, it was noted that the particle feed rate does not dramatically affect the frequency of double peaks. If particles are manually fed into the system at a very slow rate $(<0.5$ particles per second) the problem can be avoided entirely, but this defeats the purpose of the PSA. A sample lot containing roughly 6000 TRISO particles was fed through the PSA beginning with constant yet relatively slow (2-5 particles per second) rate up through the capacity of the system (Table 1). This test suggests that the fraction of double peaks observed during a run is independent of feed rate and thereby must be due to random particle trajectory perturbations initiated within the test cell inlet tube.

Table 1. Comparison of feed rates to double peak occurrence rate.

\begin{tabular}{cccc}
$\begin{array}{l}\text { Particle Feed Rate } \\
\text { (Particles/Second) }\end{array}$ & $\begin{array}{l}\text { Events } \\
\text { Triggered }\end{array}$ & $\begin{array}{l}\text { Double } \\
\text { Peaks }\end{array}$ & $\begin{array}{l}\text { Total } \\
\text { Particles }\end{array}$ \\
\hline $2-5$ & 6118 & 6 & 6124 \\
$5-10$ & 6114 & 10 & 6124 \\
$10-15$ & 6120 & 4 & 6124 \\
$16-25$ & 6115 & 9 & 6124 \\
$26+($ Max $\sim 35)$ & 6116 & 8 & 6124
\end{tabular}

If correctly identified, double peaks do not present a substantial hurdle to particle counting or sizing. Correcting the particle count when a PSA run contains a few double 
peaks is straightforward. Since each double peak is actually two particles recorded as only one, adding the number of double peak events to the total count corrects the value (as demonstrated in Table 1). Sizing a double peak is more difficult. Since the current version of the fit algorithm was developed for 'standard' single-particle events, an attempt to calculate size data from a double peak event will trigger a quality far below those seen for single peak events. As such, any double peak events are simply discarded from the sizing data. Fortunately, these outlying qualities can be used to categorize double peak events. Future development of the PSA software could easily include a modification to fit a sizing curve to a double peak. The infrequency of these events allows this omission to occur without causing significant impact to the overall shape distribution.

\section{PSA Performance}

The PSA developed at Oak Ridge National Laboratory under the AGR program is capable of rapidly processing large samples of TRISO particles at a speed of 40 particles per second with counting accuracies to within a single particle out of 10,000. This counting accuracy allows the calculation of average particle weight to an order of magnitude higher accuracy than is achieved by hand counting. It also introduces the possibility of using direct particle counting of large samples to improve or make possible other characterization methods, such as those that use porosimetry or pycnometry. In contrast, diameter values cannot be measured by the current PSA system to the level of accuracy available by the backlit optical method currently used for coated particle fuel characterization of size and shape. Figure 16 shows the particle size histograms produced by five PSA runs performed on the same sample of zirconium oxide particles. For comparison, Figure 17 shows a histogram of the same sample obtained using the shadowgraph technique. 


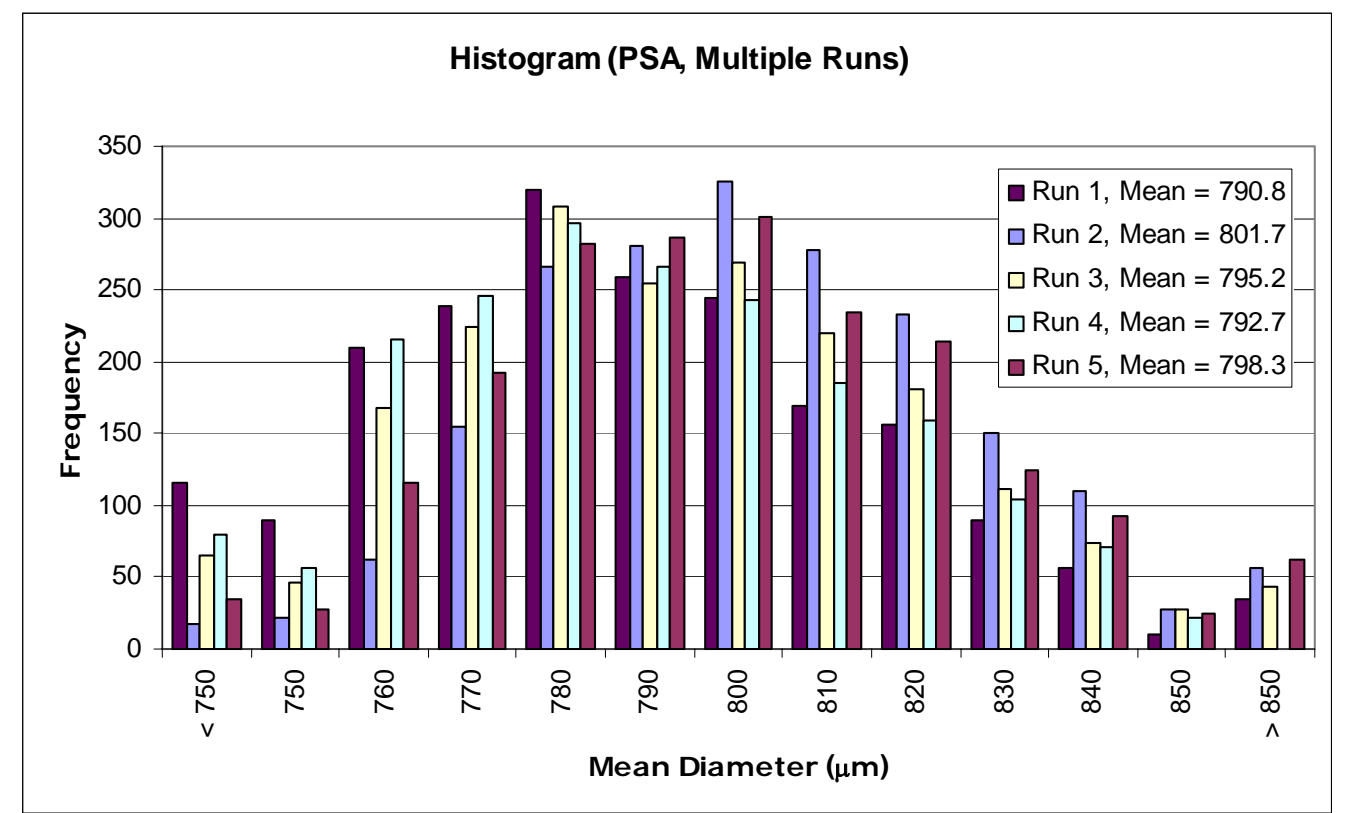

Figure 16. Histograms obtained from five separate PSA runs performed on the same sample containing roughly 2000 particles.

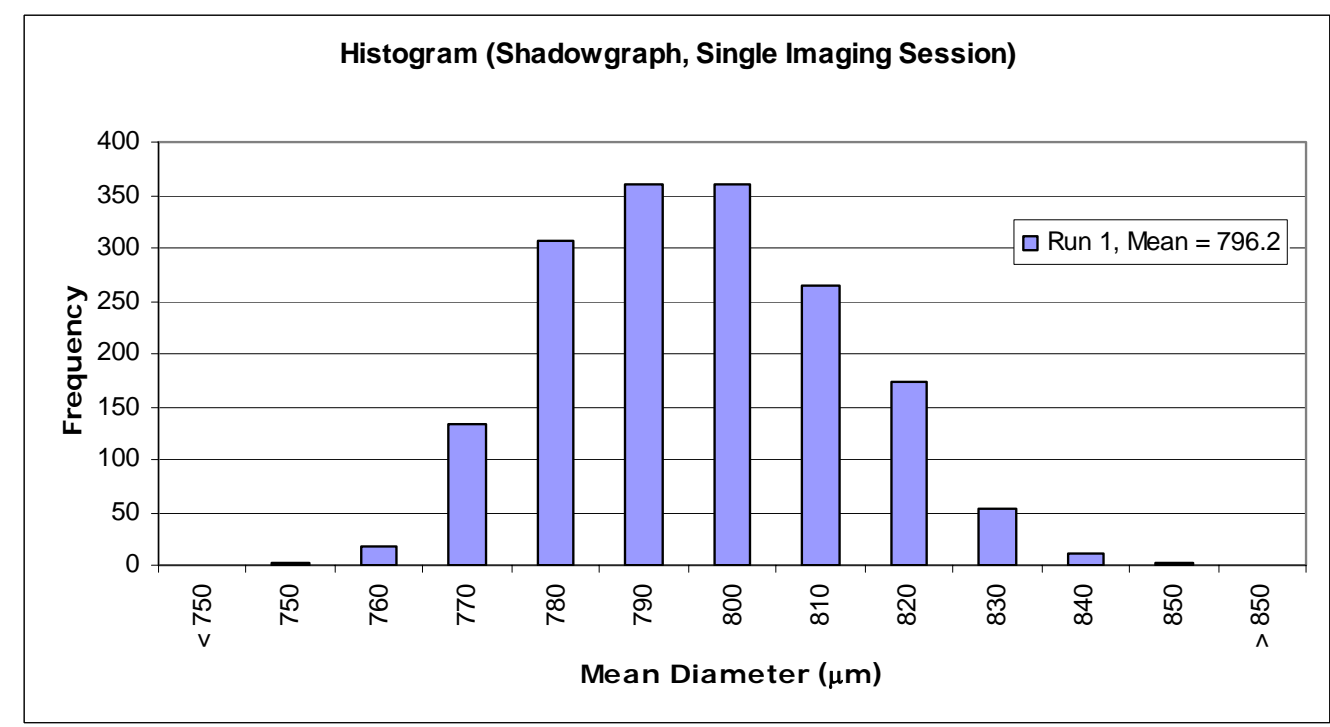

Figure 17. Histogram obtained through optical shadow imaging of roughly 2000 particles using AGR standard method.

The most obvious difference between the two histograms is that the distribution of the PSA data is broader than that obtained using the optical microscope. Both distributions center about $800 \mu \mathrm{m}$, but the PSA histogram contains a significant number of particles that are placed in the 'below-750 $\mu \mathrm{m}$ ' and 'above-850 $\mu \mathrm{m}$ ' bins. The points in these bins typically fall in a wide range $50 \mu \mathrm{m}$ below or above the cutoff. If it was determined that these data points were simply erroneous values, they could be deleted from the mean calculation. As was discussed in the software section, a method of sorting all events by examining the shape of the obscuration profile is available. Upon examining the shape of the profiles of the outlying data, a number of the events do exhibit comparatively poor 
qualities. However, this is not true of all the points. Similarly, some data near the mean region of the sample is also observed to have poor fit qualities. It is also important to recall that only the maximum obscuration value is used in determining the particle's diameter. A poor profile quality may suggest an off-normal event, but good data can still be found in events with poor profile qualities. The main use of the shape quality indicator is to separate double peaks or false events.

When these outlying regions are excluded from the calculation, the means for each run, as shown in Figure 16, are obtained. The mean diameter values produced by the PSA runs were all within $6 \mu \mathrm{m}$ of the shadowgraph result, which is comparable to the $4 \mu \mathrm{m}$ uncertainty observed when a single spherical particle was sent through the PSA multiple times. The spread in the PSA data indicates that there is larger error in the individual diameter measurements, but much of this is averaged out when calculating the mean. The variation from run to run is probably due to a combination of factors including the effect of the particle asphericity, as discussed in the next section. Further refinement in accuracy is possible when the data acquired from multiple PSA runs on a sample are combined. Averaging the runs together can reduce the measurement uncertainty introduced by such effects as particle orientation, laser intensity drift, obscuration due to soot build-up, etc. Figure 18 shows the resulting histogram when the data plotted in Figure 16 is combined together; as would be the case if the same sample was poured through the PSA five times in succession. The mean calculated from this distribution is $795.6 \mu \mathrm{m}$, nearly identical to the value provided by the shadowgraph. This offers promise that while variations within the system prevent the PSA from achieving the accuracy levels available using a shadowgraph for a single particle, repeated analysis of a suitable sample size can provide the mean diameter to high accuracy.

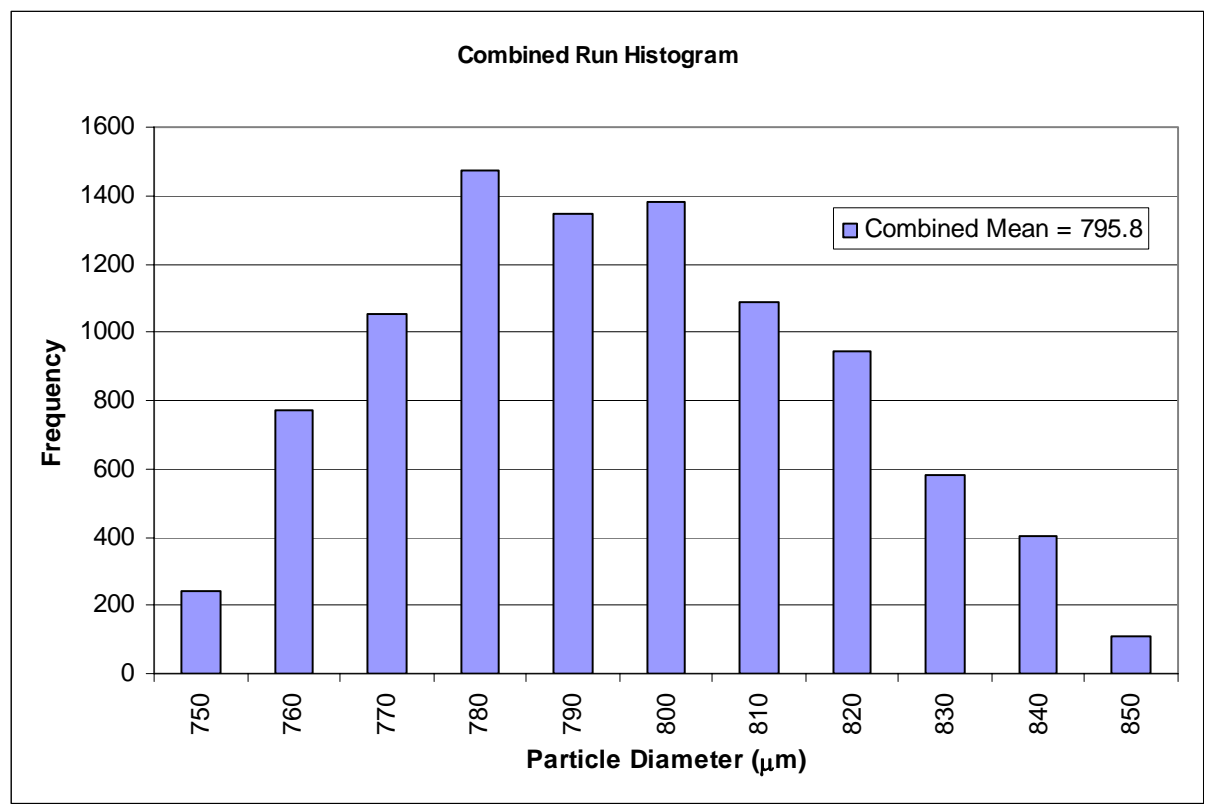

Figure 18. Histogram obtained by combining all five runs of Figure 16. Outlying data below $750 \mu \mathrm{m}$ and above $850 \mu \mathrm{m}$ has been excluded. 


\section{Effect of Particle Shape and Orientation}

While running a lot size of 2000 particles through the PSA five times has been shown to produce high quality mean data, the origin of the widened distribution needs explanation. The PSA resolution has been shown to be roughly $4 \mu \mathrm{m}$, this cannot account for the drastic increase in uncertainty for a single particle on the order of 10's of microns. Some of this could be due to asphericity in the particle shape. The shadowgraph method identifies numerous points around the perimeter of a projected particle cross section and calculates the mean diameter of that cross section. In contrast, the PSA only makes a single measurement of the cross section width at the point of greatest obscuration. This makes the PSA more sensitive to particle shape. When a sample is run through the PSA multiple times, the size measurement for each particle can be effectively obtained as a mean value, where each individual measurement is for a different random orientation of the particle. This removes much of the sensitivity of the PSA to the particle shape and may explain why the mean of the multirun PSA sample approaches the shadowgraph result after the runs are averaged together. In addition, averaging the multiple run may help average out other sources of error in the measurement. Unfortunately, since we can't track the measurement of each individual particle through the multiple pass measurement, it is not possible to obtain a mean size measurement on each particle to improve the width of the PSA size distribution. A possible future improvement for the PSA would be to use multiple lasers from different directions to obtain multiple measurements on each individual particle. This would allow some of the shape variation to be detected and averaged out.

Consideration was given to how the software data fit and associated size calculated for a particle passing though the test cell might depend on its orientation. The code analyzes a particle profile and produces a size estimate based primarily on the depth of the profile. As the particle shape deviates more and more from a perfect sphere, the profile shape becomes more strongly related to the orientation of the particle as it passes through the line laser. An exaggeration of this is shown in Figure 19. When a very misshapen particle passes vertically though the test cell, a wide shallow profile is produced. In the opposite situation, a narrow deep profile will be generated. Clearly, this result will introduce large errors into the lot size estimate if it can not be corrected. 


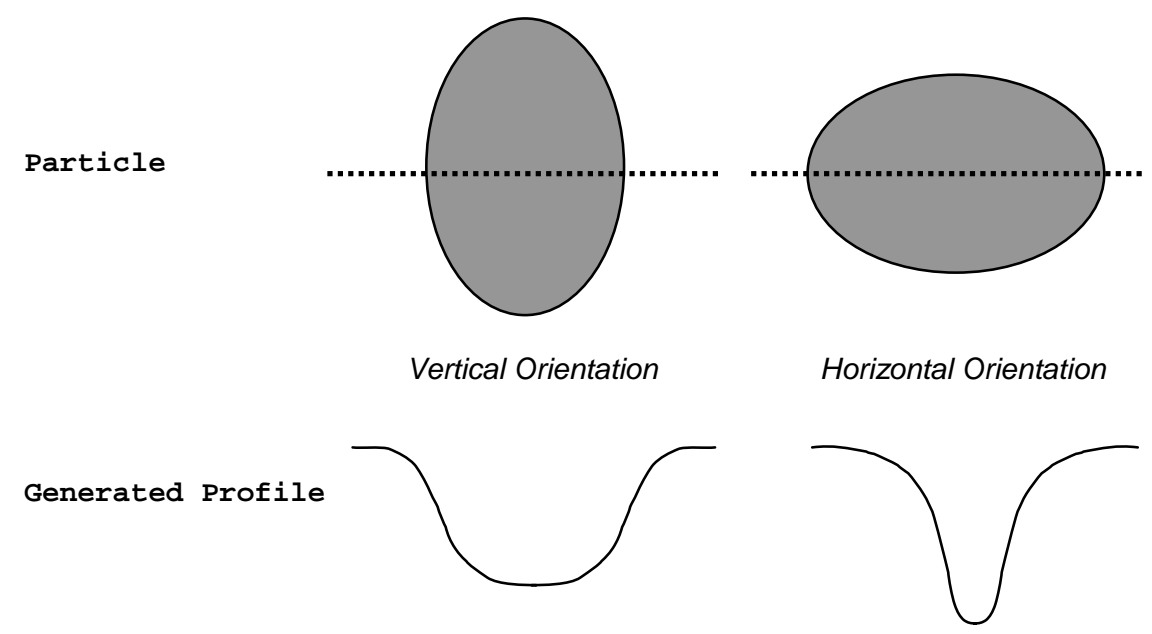

Figure 19. Comparison of profiles generated by the same particle with different orientations.

The first step taken in addressing the particle orientation error was investigation of the phenomenon to determine if it could indeed account for the poorer PSA sizing performance. The range and distribution of TRISO shapes has been well-documented through use of optical microscopy. While it is clear that particles with highly elliptical cross sections will incur significant sizing errors depending on their orientation through the PSA, it may be that the small variations between the major and minor axes found in TRISO fuel cross sections will not. The Matlab software package was used to write an algorithm to model the light blocked by a particle with an elliptical cross section falling through a $20 \mu \mathrm{m}$ thick laser line, where the particle orientation could be varied. Five random orientations are plotted in Figure 20. An $800 \mu \mathrm{m}$ particle was modeled with an aspect ratio of 1.05, as these are typical means of TRISO fuel sample lots. It is apparent that even this comparatively sphere-like shape will result in errors in diameter estimation using the maximum obstructed light as a predictor. In the five examples below, the maximum particle width passing through the line laser at one time ranged from 781 to $813 \mu \mathrm{m}$. Since the fitting algorithm assumes a spherical shape, these values would be recorded as the particle's diameter. 


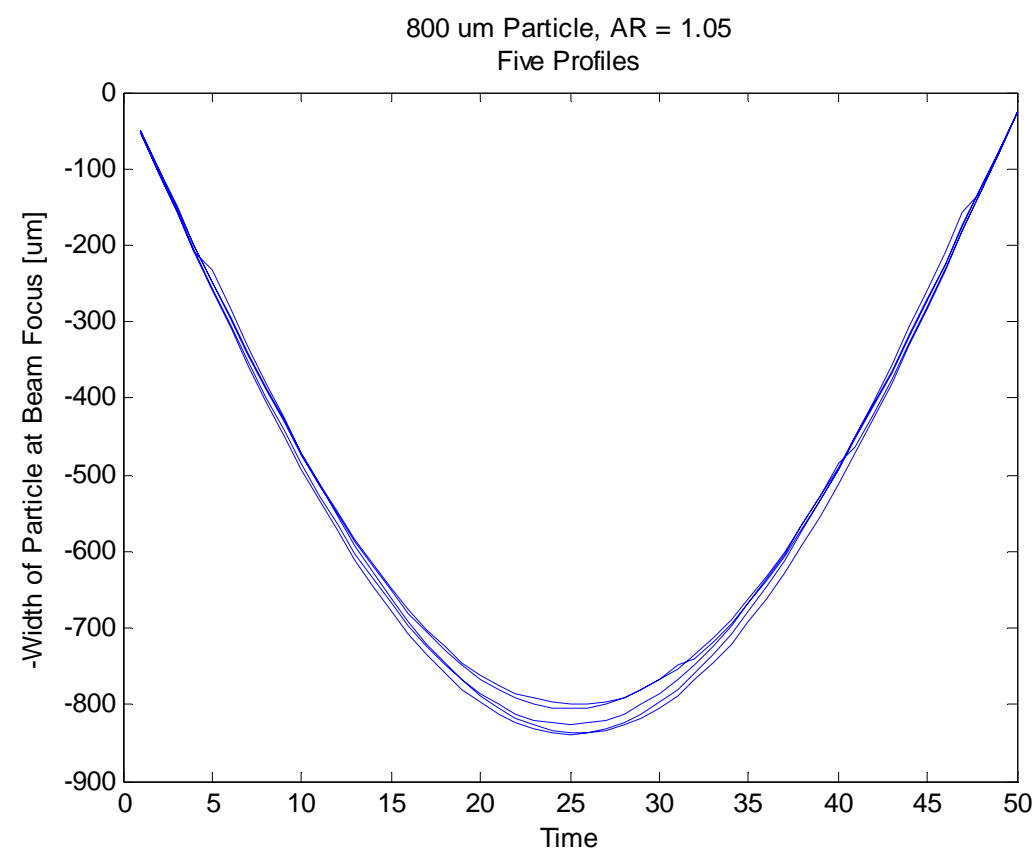

Figure 20. Five profiles generated by an $800 \mu \mathrm{m}$ particle with an aspect ratio of 1.05 (820 $\mu \mathrm{m}$ major axis, $780 \mu \mathrm{m}$ minor axis) passing through the test cell simulated in Matlab. The $y$-axis is plotted as the negative of the particle width at the beam focus since this correlates directly to the amount of light detected by the photoreceiver. The $x$-axis is unitless since the sampling rate used in Matlab has no relation to any physical quantity.

Using the same numerical code, 500 trials were run with random orientations on the same shaped particle (820 $\mu \mathrm{m}$ major axis, $780 \mu \mathrm{m}$ minor axis). The histogram generated by this test is shown in Figure 21. The intuitive result shown here suggests that an equal number of particles will fall through the line laser with a horizontal orientation as a vertical orientation, as well as the intermediate degrees of off-axis rotation. The overall result of this phenomenon will be a broadening of the diameter distribution, since now there are a large number of particles detected with extreme diameters, but the mean will remain roughly the same; indeed, the mean calculated by the 500 particles in the trial below was found to be $799.3 \mu \mathrm{m}$. 


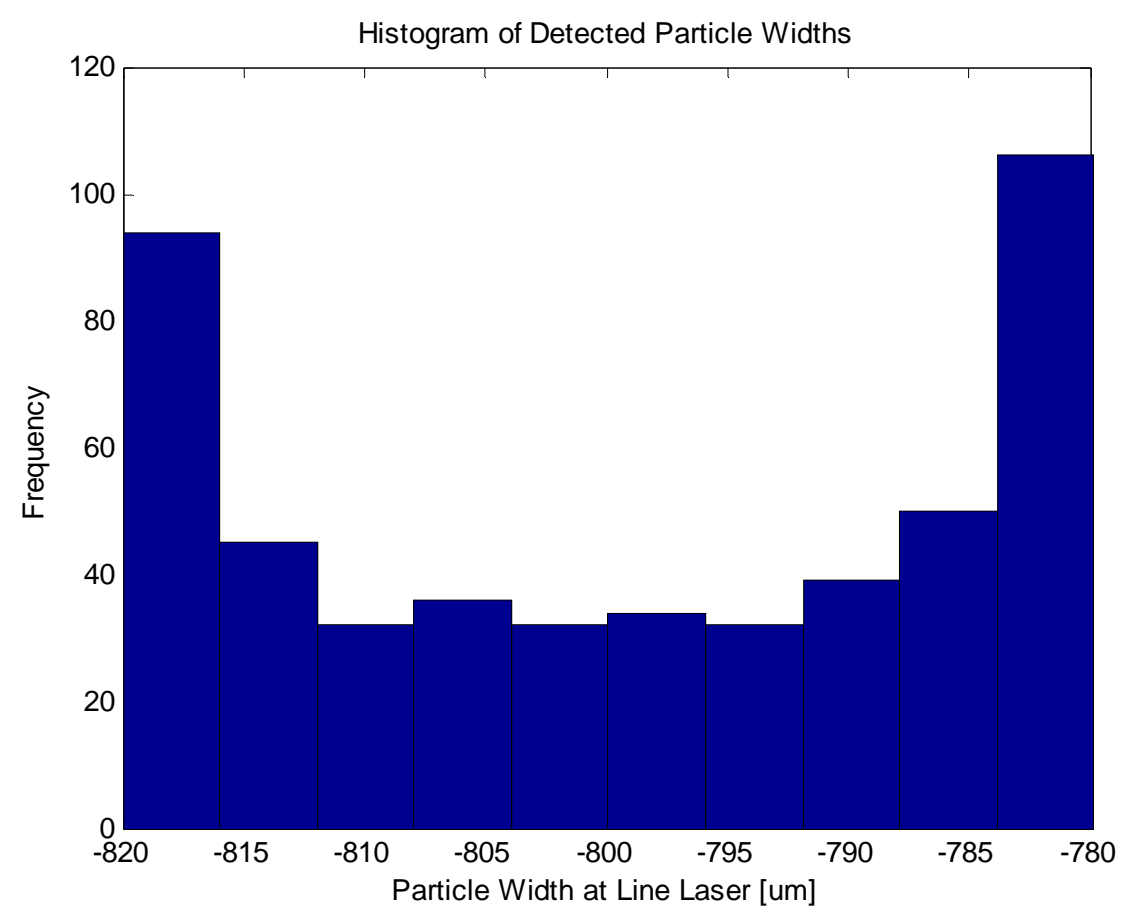

Figure 21. Histogram of particle widths recorded by $800 \mu \mathrm{m}$ particle with an aspect ratio of 1.05 passing through the test cell simulated in Matlab. 500 trials were run with random particle orientations.

Given this result, the behavior observed in Figure 16 and Figure 17 can be understood. The histograms constructed using the PSA analysis had much wider distributions compared to the one obtained using shadow imaging. However, the sample mean calculated from four of the five trials agreed with the accepted mean within a few microns. This can be attributed to the addition of outlying particle diameters equally at both ends of the distribution that are in reality the more elongated particles of the lot passing through the line laser at extreme horizontal or vertical orientations. While this is acceptable when only the mean diameter of a sample is of interest, it will prevent the PSA from successfully calculating a particle's shape unless the software algorithm is improved in a manner such that the particle's orientation can be discerned from its profile. Unfortunately, this means that the particle's speed will become a necessary variable within the analysis.

Relating the particle profile to its shape would be possible if a particle's speed was a known quantity. In the Matlab analysis performed above, a wide, shallow profile corresponds to a vertical orientation while a narrow, deep profile is evidence of a horizontal orientation. With a proper fit algorithm, the orientation and in turn sizing data could be extracted. However, the simulation samples points at a constant rate from one end of the particle to the other. The photoreceiver also samples at a constant rate, but the linear distance traversed between data points will be a function of the particle's velocity. If one event has a width much greater than the next, it might mean that the first simply had a much slower velocity through the test cell compared to the second opposed to different orientations of an elliptical particle. 


\section{Particle Speed}

Calculating an individual particle's speed inside the PSA was never a stated design objective. The particle's transit time was of indirect interest, since one of the stated goals of the PSA was a capability to rapidly process large particle samples; the notable difference being that time required for processing of an entire sample varies greatly from controls placed on a particular particle velocity.

Although it has not been attempted to date, substituting a transparent particle accumulator for the current metal variant at the bottom of the cyclone separator might allow for crude timing of individual particle's transit through the entire system. However, the system transit times will likely have little relation to the time a particle spends within the cell. The velocity of an individual particle will also be a function of the instantaneous negative pressure that is affected by the number of particles currently within the system.

Additional lasers placed at locations within the cell might allow a measurement of transit times within the cell itself, but would require extensive modification to nearly all aspects of the current PSA design. As such, it is unlikely that the current generation PSA will be able to incorporate a particle velocity measurement and implementation into sizing efforts.

One additional benefit that would be available should particle velocities be provided with considerable accuracy will be the freedom to move to a more advanced type of event analysis with regard to extracting particle diameter. In the original PSA development paper, where the mathematical basis for calculating a particle's diameter based upon its obscuration profile is discussed ${ }^{4}$, three techniques are outlined. Two of the three are discarded immediately as they include the particle velocity as an independent variable. With the inclusion of a velocity term, these methods might allow a higher degree of accuracy in both size and shape to be obtained.

\section{Conclusions}

A PSA was successfully developed that is capable of counting TRISO particles with an uncertainty of less than one particle per 10,000 counted. This can be accomplished at a rate of 35 particles per second. Particle sizing is also possible within $4 \mu \mathrm{m}$ for spherical particles, but the characterization metric is less successful when individual aspherical particles are analyzed. Error in aspherical particle size measurement can be averaged out using multiple passes through the PSA. The mean diameter of a particle lot determined using multiple sample runs agrees well with the diameter data determined using standard shadowgraph techniques. However, this averaging method only improves the mean diameter measurement. The standard deviation of the distribution is still effected by the asphericity error in each individual particle measured. Further testing of particle shapes and lot sizes is needed to determine more specifically the exact capabilities and uncertainties of the PSA analysis. A more precise means of characterizing event profiles 
would aid in evaluating outlying data as well as assigning confidence to specific points. Potentially, this could be adapted to a method of calculating a particle's aspect ratio. 


\section{References}

[1] J.R. Price and J.D. Hunn, "Optical inspection of coated particle nuclear fuel," in Proceedings of Machine Vision Applications in Industrial Inspection

XII, SPIE Vol. 5303, 2004, 137-149.

[2] J.R. Price, D.B. Aykac, J.D. Hunn, A.K. Kercher, and R.N. Morris "New developments in image-based characterization of coated particle nuclear fuel," Proceedings of Machine Vision Applications in Industrial Inspection XIV, SPIE Vol. 6070, 2006, 153-162.

[3] K. Wallisch and P. Koss, "Automatic Size Analysis of Coated Fuel Particles,” Nuclear Technology, 35, 279 (1977).

[4] T. Karnowski, A. Kercher, J. Hunn, and C. Maxey, "A simple optical system for realtime measurements of TRISO fuel pellets," Machine Vision Applications in Industrial Inspection XIII, 5679, 62 (2005). 